\title{
Foreword
}

\section{Thematic Section: Research with the child, the mother, and the family}

We are pleased to present another thematic section in our journal. This is an initiative that harmonizes the pursuit of scientific knowledge with different areas of interest, weaving the articles according to a common thread of meaning. Although studies on childhood, maternity, and family are common in psychological research, the social transformations of the recent decades indicate the need for changes in the focus or methods in order for science to serve the purpose of understanding a reality, a phenomenon, or a situation. The efforts made by the authors of the studies presented here, lead us to believe that psychology research has undergone fundamental changes, as indicated by the topics covered, which are in tune with the new demands of society.

Francischini and Fernandes address the contentious issue of ethical research with children based on a search conducted on European databases to identify articles published over the last two decades. The lack of studies on research ethics demonstrate the need to devote more attention to the participatory space of children in research, giving them a voice and the right to make decisions, together with their parents, about their participation or about the use of more interactive research methods, which are therefore more compatible with the way children express themselves.

Aching and Granato draw an interesting parallel between the Winnicott's good-enough mother, a concept of adequate mothering for the development of children's potential, and the views of pregnant women and mothers of what it means to be a good mother in situations of social vulnerability. The authors discuss the conditions of being a mother in a precarious social context, in which not only deprivation, loneliness, and prejudice, but also hope for a better life and personal fulfillment nurture a singular mother-infant relationship.

Focusing on presenting methodological alternatives for qualitative research in psychoanalysis, Granato and Aiello-Vaisberg propose the use of Interactive Narratives as an approach that is expressive, playful, and conformable to life experiences. To illustrate the use of this dialogical procedure, the authors invite undergraduate students to complete a fictional story about a couple which faces the possibility of having a child with Down syndrome and discuss the narrative material in terms of the different conceptions of motherhood and possible ways to resolve their conflicts. Moreover, the authors discuss the issue of motherhood seen as choice or as a destiny for women, which entails expectations about the maternal figure.

As a counterpoint to motherhood in a context of social precariousness, there is the study of Pessôa et al., who evaluate three different dyads composed of caregivers in a middle class context - mothers and grandmothers, mothers and nannies, and mothers and daycare educators -, in order to investigate their views of child care. The authors adopted an innovative approach to evaluate different discourses using photographs of mothers and their babies. They found that the participants tended to focus on physical contact, autonomy, affection, and security, among other elements involved in child care.

Canosa and Postalli, on the other hand, analyze domestic routines of bathing and feeding in order to observe the gentle communication established between the mother and her blind baby. Maternal sensitivity 
is increased in the context of visual impairment in order to understand, stimulate, communicate, and facilitate the development of the child's potential. This context also emphasizes human creativity to cope with adversities, making them both an opportunity for mother's growth and an early stimulation of the child, as stressed by the authors.

In contrast, the study of Cézar and Smeha shows how autism spectrum disorders exert an indelible impact on a family. The authors addressed this issue from the perspective of adults with autistic siblings. Having to take on a parent role, they did not have much time to play, compete, or even fight with their siblings, and therefore their relationship was based on protection and care for the autistic sibling to help the overburdened mother. We hope that advances in early diagnosis of autism and approaches to caregiving that involve the whole family may, besides contributing to the development of autistic children, help these families overcome loneliness and isolation.

Finally, Henriques et al. investigate the intriguing relationship between parents and young adults who had not left parental home, regardless of their financial situation, a growing trend in today's modern society, especially among the Brazilian middle class. Marked by ambiguous discourses, which suggest intense feelings of ambivalence in this type of family configuration, according to the authors, leaving home has been a challenge for young adults as much as it is to stay longer than socially expected. Parents and children have to face the difficult task of rebuilding their relationship in an even-handed fashion, which certainly causes discomfort and demand negotiations.

In conclusion, we hope we have contributed by bringing together studies addressing similar issues, concerns, or dilemmas that motivate us to search for answers, which, in addition to providing understanding or explanations, aim to provide knowledge that improves the quality of people's life. Happy reading everyone!

Tania Mara Marques Granato

Associate Editor

Pontifícia Universidade Católica de Campinas,

Centro de Ciências da Vida, Programa de Pós-Graduação em Psicologia como Profissão e Ciência 